\title{
Water vapor therapy (Rezūm) for lower urinary tract symptoms related to benign prostatic hyperplasia: early results from the first Italian multicentric study
}

\author{
Giampaolo Siena ${ }^{1} \cdot$ L. Cindolo ${ }^{2,3} \cdot$ G. Ferrari ${ }^{3} \cdot$ D. Maruzzi ${ }^{4} \cdot$ G. Fasolis ${ }^{5} \cdot$ S. V. Condorelli ${ }^{6} \cdot$ F. Varvello $^{5} \cdot$ F. Visalli $^{4}$. \\ S. Rabito ${ }^{3}$-S. Toso $^{3} \cdot$ S. Caroassai ${ }^{1}$ - A. Mari ${ }^{1}$ - L. Viola ${ }^{1}$ - B. K. Somani ${ }^{7} \cdot$ M. Carini $^{1}$
}

Received: 23 November 2020 / Accepted: 18 February 2021 / Published online: 31 March 2021

(c) The Author(s), under exclusive licence to Springer-Verlag GmbH Germany, part of Springer Nature 2021

\begin{abstract}
Purpose Rezūm is the latest developed minimally invasive treatment for benign prostatic hyperplasia (BPH). We aimed to carefully assess the functional outcomes of patients treated with Rezūm for BPH.

Methods We prospectively followed 135 consecutive patients treated by Rezūm at 5 institutions from June 2019 to August 2020. The International Prostate Symptom Score (IPSS), International Consultation on Incontinence Questionnaire-Short Form (ICIQ-UI SF), the Overactive Bladder Questionnaire-Short Form (OAB-q SF) score, the International Index of Erectile Function (IIEF-5) and questions 9 and 10 to assess ejaculatory dysfunction were recorded.

Election criteria were age $>18$, no prior prostate interventions, IPSS $\geq 13$, post-void residual $\leq 250 \mathrm{~mL}$, prostate volume between 30 and $120 \mathrm{cc}$.

Results The median operative time was 10.5 (IQR 8.7-15) min. All patients were dismissed few hours after surgery with indwelling urinary catheter that was removed after a median of 7 (IQR 7-10) days. A significantly decrease of IPSS from baseline at first $(p=0.001)$ and third $(p<0.0001)$ month after surgery was reported. No difference was reported in terms of ICIQ-UI SF score postoperatively. A mild reduction of the OAB-q SF score was reported at 1 month from surgery $(p=0.06)$ that turned significant at 3 months postoperatively $(p<0.0001)$. A slight but statistically significant increase of the IIEF-5 score was reported from baseline at 6 months $(p=0.04)$. Postoperatively, patients reported a significantly decrease of ejaculatory dysfunction after alpha-blocker interruption.

Conclusion Rezūm treatment is a feasible minimally invasive option for patients with BPH symptoms and showed optimal early functional outcomes.
\end{abstract}

Keywords Benign prostatic hyperplasia $\cdot$ Rezūm $\cdot$ Minimally invasive

\section{Introduction}

It is estimated that BPH currently affects $6 \%$ of the male population worldwide $[2,3]$. Symptoms related to $\mathrm{BPH}$ increases proportionally with the age [1-3]. There is

Giampaolo Siena

giampaolo.siena@gmail.com

1 Department of Urology, Careggi Hospital, University of Florence, San Luca Nuovo, Florence, Italy

2 Department of Urology, Villa Stuart, Private Hospital, Rome, Italy

3 Department of Urology, Hesperia Hospital, Cure Group, Modena, Italy evidence from longitudinal studies that $\mathrm{BPH}$ is a progressive disease: prostate size increases, symptoms and health-related quality of life worsen, flow rates deteriorate, and some men develop unfavorable outcomes such as acute urinary retention (AUR), or the need for surgery [4]. Several options exist
4 Department of Urology, S. Maria Degli Angeli Hospital, Pordenone, Italy

5 Department of Urology, Michele e Pietro Ferrero Hospital, Alba-Bra, Italy

6 Department of Urology, Vittorio Emanuele Hospital, Gela, Italy

7 University Hospital Southampton NHS Trust, Tremona Road, Southampton SO16 6YD, Hampshire, UK 
for BPH management with a significant range of invasiveness, efficacy, and cost [5, 6].

Decision making varies according to severity of symptoms, patients' interest in sexual function preservation and clinical features such as: prostatic length and volume (PV), prostatic urethral angle (PUA), median lobe (ML), elevated central zone (ECZ), intravesical prostatic protrusion (IPP) patients' pain tolerance and propensity to bleeding [5-8]. Minimally invasive surgical treatments (MISTs), both based on physical energy and mechanical expander options, represent alternative intervention before or after any pharmacotherapy [9-14].

The latest developed MIST is water vapor thermal therapy using radiofrequency to create thermal energy (Rezūm System, Boston Scientific, Marlborough, MA) in the form of water vapor [15-17].

The Rezūm treatment can be quickly performed under local anesthesia in an office setting. Steam is delivered to the hyperplastic prostatic tissue through a dedicated cystoscope for a short amount of time ( $9 \mathrm{~s}$ for each injection) leading to cell membrane disruption [15-17]. Within 3 months the treated tissue shrinks up to $40 \%$ and patients report relief of LUTS and enhanced quality of life without sexual function disturbance [15]. All zones of the prostate are amenable for treatment, including intravesical median lobe [7]. The aim of this study is to present a detailed prospectively collected record of the functional outcomes of patients treated with Rezūm for benign prostatic hyperplasia.

\section{Materials and methods}

Between June 2019 and August 2020, patients with moderate-to-severe lower urinary tract symptoms (LUTS) were screened in five different institutions. A standard pre-operative protocol that included the epidemiological and clinical features of the patients, digital rectal examination, serum prostate-specific antigen levels (PSA), complete blood count and chemistries, urinalysis and culture, was performed.

The International Prostate Symptom Score (IPSS), International Consultation on Incontinence Questionnaire-Short Form (ICIQ-UI SF), the Overactive Bladder QuestionnaireShort Form (OAB-q SF) score, the International Index of Erectile Function (IIEF-5) and two further question of the IIEF-15 questionnaire (Questions 9: "over the past 4 weeks, when you had sexual stimulation or intercourse how often did you ejaculate?" and Question 10 "over the past 4 weeks, when you had sexual stimulation or intercourse how often did you have the feeling of orgasm or climax with or without ejaculation?" were self-administered and collected preoperatively and during the follow-up. Finally, uroflowmetry and an ultrasonography to measure the prostate volume and post-voiding residual volume were checked pre-operatively and during the follow-up.

Election criteria were age $>18$ years, no prior interventions for BPH, International Prostate Symptom Score (IPSS) $\geq 13$, peak urinary flow rate $($ Qmax $) \leq 15 \mathrm{~mL} / \mathrm{sec}$ with minimum voided volume of $\geq 125 \mathrm{~mL}$, post-void residual $\leq 250 \mathrm{~mL}$, prostate volume $>30$ and $\leq 120 \mathrm{cc}$.

The first 135 consecutive patients treated with the transurethral intraprostatic water vapor injections (Rezūm System, Boston Scientific, Marlborough, MA) at five institutions were prospectively followed.

\section{The Rezūm procedure}

Patients were placed in a lithotomy position. The instrument was inserted into the urethra to access the transition zone of the prostate with the water vapor delivery device. The polyether-ether ketone (PEEK) vapor needle under direct visualization penetrated into the prostate, and water vapor was circumferentially dispersed around the tissue for a duration of $9 \mathrm{~s}$. Heat dispersed rapidly throughout the adenoma and the quick increase of the temperature to approximately $70{ }^{\circ} \mathrm{C}$ led to cell death.

The injections were performed $1 \mathrm{~cm}$ under the bladder neck and caudally down the length of the prostatic urethra to the proximal edge of the verumontanum each centimeter. When present, the median lobe was treated with 1 or more injections. The number of water vapor injections depended on the volume of the prostate, presence of the median lobe and the length of the prostatic urethra. The exact number of injections was calculated with the help of a view finder placed at the tip of the instrument that measures the field of view (FoV) of $0.5 \mathrm{~cm}$ (Fig. 1). Therefore, it is possible to estimate the exact urethral length while retracting the instrument from the bladder neck back to the verumontanum.

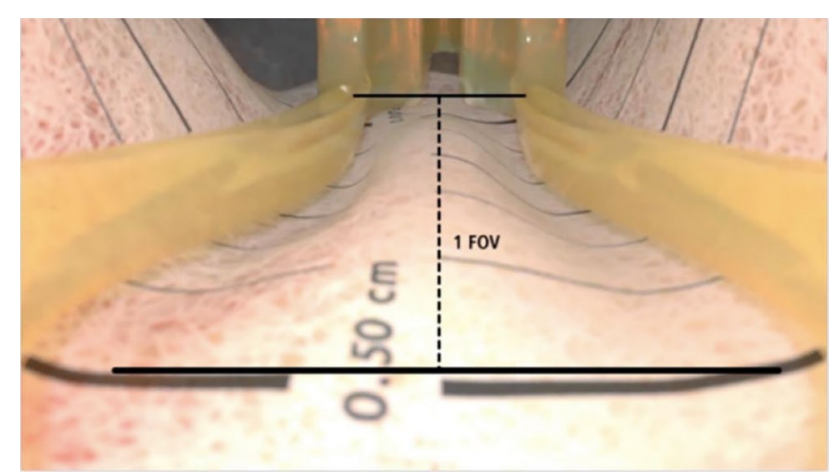

Fig. 1 a Transurethral insertion of the Rezūm device. b The polyether-ether ketone (PEEK) vapor needle is inserted under direct visualization into the prostate, and water vapor was circumferentially dispersed around the tissue for a duration of $9 \mathrm{~s}$ per injection. $\mathbf{c}$ Removal of the needle from the prostate without any local bleeding or immediate effect on the tissue 
Approximatively, a steam injection was done each $1 \mathrm{~cm}$ : at 8 and 4 o'clock on lateral lobes and at 6 o'clock on median lobe.

The use and type of anesthesia were variable from oral sedation to prostate block, intravenous sedation or mild general anesthesia in accordance with local protocol and patients' preferences. Antibiotics were administered to all patients according to local practice guidelines. Prophylaxis included either Quinolones (Levofloxacin $500 \mathrm{mg}$ once daily for 7 days) or Cephalosporine $400 \mathrm{mg}$ daily for 7 days.

\section{Statistical analyses}

Continuous parametric variables were reported as median and interquartile range (IQR). Categorical variables were reported as frequencies and proportions. The Student's paired $t$ tests were used to compare two dependent factors. Statistical significance was set as $p<0.05$. All tests were two-sided. Analyses were performed using STATA v.14.1 (StataCorp LP, College Station, TX), graphics using Microsoft ${ }^{\circledR}$ Excel Professional Plus 2016.

\section{Results}

Overall, 135 patients were enrolled and their outcomes analyzed. The pre-operative characteristics are summarized in Table 1. Patients had a median age of 69 (interquartile range [IQR] 61-79) years, a median PSA of 2.1 (IQR 1.3-4.0) ng/
$\mathrm{mL}$ and a PV of 60 (IQR 45-78) mL. At uroflowmetry, the preoperative median Qmax and Qmed were 8.1 (IQR 6-10) and 3.7 (IQR 2.1-6.2). Before surgery all patients received treatment with alpha-blockers for a median time of 28 (IQR 10-42) months. In addition, 4 (3\%) received Serenoa Repens alone and 4 (3\%) 5-alpha reductase inhibitors. Ten $(7.4 \%)$ patients had acute urinary retention (AUR) and had indwelling catheter. The pre-operative median total IPSS was 21.5 (IQR 17-25). The ICIQ-UI SF score was below $1 / 21$ in all patients. The pre-operative median OAB-q SF score was 33 (IQR 19-52) over 78 and the median IIEF-5 score was 21.5 (IQR 17-25).

The median operative time from the instrument transurethral insertion to patient catheterization was 10.5 (IQR 8.7-15.0) minutes. Patients received a median of 7 (IQR 5-8) PEEK vapor needle injections. A 22Ch urinary catheter was positioned in the first three cases, while in the following procedures due the absence of postoperative macrohematuria either a $18 \mathrm{Ch}$ urinary catheter or a temporary prostatic stent (Exime ${ }^{\circledR}$, Rocamed) that allows complete urinary continence and spontaneous urination were placed. Postoperative outcomes are reported in Table 2. All patients were dismissed few hours after surgery with indwelling urinary catheter that was removed after a median of 7 (IQR 7-10) days. Complication rate was $48 \%$, all grade 1 according to Clavien-Dindo classification. Complications were: mild hematuria, hematospermia, dysuria, urinary tract infections (UTI) and AUR. Mild hematuria, hematospermia and dysuria were self-limiting within maximum 6 weeks. UTI
Table 1 Pre-operative characteristics of 135 patients treated with Rezūm system

\begin{tabular}{lll}
\hline Preoperative characteristics $(n=135)$ & & \\
\hline Age $($ years), median IQR & 69 & $61-79$ \\
BMI $\left(\mathrm{kg} / \mathrm{m}^{2}\right)$, median IQR & 24 & $22.8-26$ \\
ASA PS score, median IQR & 2 & $1-2$ \\
Preoperative median uroflowmetry Qmax $(\mathrm{ml} / \mathrm{s})$, median IQR & 8.1 & $6-10$ \\
Preoperative median uroflowmetry Qmed $(\mathrm{ml} / \mathrm{s})$, median IQR & 3.7 & $2.1-6.2$ \\
Preoperative medical treatment for BPH $n$. $\%]$ & & \\
Alpha-blocker & 66 & $48.9 \%$ \\
5-ARI & 4 & $3 \%$ \\
Phytotherapic & 4 & $3 \%$ \\
Alpha-blocker, 5-ARI, phytotherapic & 23 & $17 \%$ \\
Alpha-blocker, 5-ARI & 33 & $24.4 \%$ \\
Alpha blocker, phytotherapic & 4 & $3 \%$ \\
Anticholinergic & 1 & $0.74 \%$ \\
Pre-operative IPSS score, median IQR & 21.5 & $17-25$ \\
Pre-operative ICIQ-UI SF score, median IQR & 0 & $0-1$ \\
Pre-operative OAB-q SF score, median IQR & 33 & $19-52$ \\
Pre-operative IIEF score, median IQR & 20 & $16-22$ \\
\hline
\end{tabular}

IPSS International Prostatic Symptoms Score, ICIQ-UI SF International Consultation on Incontinence Questionnaire-Urinary Incontinence Short Form, $O A B-q S F$ Overactive Bladder Questionnaire Short Form, IIEF International Index of Erectile Function 
Table 2 Postoperative characteristics of 135 patients treated with Rezūm system at 1, 3 and 6 months follow-up

\begin{tabular}{lll}
\hline Postoperative outcomes $(n=135)$ & & \\
\hline Postoperative acute urinary retention $[n . \%]$ & 16 & $11.8 \%$ \\
Day of urinary catheter removal (median IQR) & 7 & $7-10$ \\
1st month IPSS score (median IQR) & 7.5 & $5-12$ \\
3rd month IPSS score (median IQR) & 4.2 & $3.2-5.3$ \\
6th month IPSS score (median IQR) & 4.4 & $3.8-5.9$ \\
1st month OAB-q SF score (median IQR) & 16.5 & $13-3 .-23.7$ \\
3rd month OAB-q SF score (median IQR) & 16 & $14.5-16.4$ \\
3rd month IIEF-5 score (median IQR) & 12.5 & $0.7-21.7$ \\
6th month IIEF-5 score (median IQR) & 23.5 & $21-25.5$ \\
\hline
\end{tabular}

occurred in eight patients (6\% of the cases) and required to prolong the antibiotics. Sixteen $(11.8 \%)$ patients had acute urinary retention (AUR) at catheter removal at 7 th postoperative day. They were treated with indwelling catheter for additional days, median 3 more days (IQR 1-21). No statistical differences were found between the subgroup of patients who had AUR at catheter removal and the subgroup who did not, in terms of pre-operative PV, PVR, Qmax, presence of median lobe $(p<0.001)$. BPH medication was interrupted 2 weeks after catheter removal.

Three patients required definitive TURP: patient 1 had a pre-operative AUR, PV of $83 \mathrm{cc}$; patient 2 had PV $78 \mathrm{cc}$, IPP grade 2, PVR $200 \mathrm{cc}$; patient 3 had a Qmax 7.6 Ml/sec; PVR $400 \mathrm{cc} ;$ PV $25 \mathrm{cc}$. Median PV at 6 months postoperatively was 54 (IQR 39-68) mL.

Patients reported a significantly decrease of IPSS from baseline at first [21.5 (IQR 17-25) vs 7.5 (5-12.), $p=0.001]$, third [21.5 vs 4.2 (IQR 3.2-5.3), $p<0.0001]$ and sixth [21.5 vs 4.4 (IQR 3.8-5.9), $p<0.0001$ ] months after surgery. No difference was reported in terms of ICIQ-UI SF score postoperatively. A mild reduction of the OAB-q SF score was reported at 1 month from surgery [33 (IQR 19-52) vs 16.5 (13.3-23.7), $p=0.06]$ that turned significant at 3 months postoperatively [33 vs 13 (IQR 12.5-16.4), $p<0.0001$ ]. A slight but statistically significant increase of the IIEF-5 score was reported from baseline at 6 months [20 vs 23.5 (IQR $21-25.5), p=0.04]$. Postoperatively, a scoring of 4 and 5 at Question 9 of the IIEF-15 questionnaire was reported in 51 $(81 \%)$ and $11(17.1 \%)$ of cases. A scoring of 5 at Question 10 of the IIEF-15 questionnaire in $98.5 \%$ of cases $(p<0.0001$ compared to pre-operative results in both cases).

\section{Discussion}

In this study, we presented the midterm functional outcomes in patients treated with the Rezūm System, the latest developed MIST using radiofrequency to thermal energy in the form of water vapor leading to tissue shrinks up to $40 \%$ [15]. The debulking effect (anatomical effect) was clearly evident at postoperative cystoscopy and it is in line with previous study on Magnetic Resonance Imaging (MRI) that reported one-third reduction in overall prostate and transition zone volumes [16]. Our study showed a significant decrease in the IPSS scores at first and third month after surgery consistently with previous studies $[6,15]$. Herein, a detailed collection of postoperative symptoms was done through validated patient-reported scores. No de novo postoperative incontinence was reported. Moreover, a mild reduction of the storage symptoms was reported after one month and symptoms progressively disappeared at three months after the procedure. Rezūm treatment is a promising procedure. In contrast to some of the other MIST such as Urolift and urethral stents, Rezūm can be also used for enlarged median lobes [18]. In contrast to Aquablation and as prostate artery embolization (PAE), the Rezūm system has demonstrated to be successful in the day-case setting and this is relevant in the COVID-19 era, where the need to further reduce theater time and hospital stay is extremely important [19-23]. Moreover, the treatment time is short with procedures performed in office or outpatient setting thereby reducing the overall cost allowing high patient turnover. Although prostate size is a theoretical limitation of this procedure, reports have shown that it can be performed in larger prostates too. The reported complications are minor and usually self-limiting resolving within a few weeks. From zero to $2 \%$ of retrograde ejaculation and no de novo erectile dysfunction are reported after Rezūm treatment, in line with other MISTs technique such as Urolift [24]. Preservation of sexual function is seen as a clear advantage to TURP. In our study, a clinically mild but statistically significant increase of IIEF-5 in patients after the treatment was reported. This could be related to the reduction of the urinary symptoms and the better quality of life leading to more satisfying intercourses. Moreover, all but one patient recovered their ejaculatory function after Rezūm treatment and alpha-blocker interruption. Therefore, Rezūm seems to outperform the other MIST procedures and medical treatments regarding the sexual outcomes. Indeed, a scoring of 3/4 at Question 9 of the IIEF-15 questionnaire was reported in $81 \%$ of cases. Although in these cases ejaculation was present, patients sporadically reported a minor volume and a more translucent appearance of the seminal fluid.

A scoring of 5 at Question 10 of the IIEF-15 questionnaire in $98.5 \%$ of cases $(p<0.0001$ compared to pre-operative results in both cases).

Special considerations should be done on catheterization after the procedure. In the literature, catheterization time after Rezūm treatment ranges between 0 and 4 days based on each clinical case [7]. In our series, we opted for a prudentially longer catheterization time to minimize AUR that 
is a likely to happen but easily resolvable. In our multicentric series, AUR occurred in $16(11.8 \%)$ cases at catheter removal. Of those, all (81\%) but three cases were successfully treated with indwelling catheter for a median of additional 3 days (1-30). These three cases required a definitive surgery (i.e. TURP). This is in line with McVary pivotal study on Rezūm that reports $2.2 \%$ of retreatment rates at 1-year follow-up [7]. In the present series, we investigated the option of a special temporary prostatic stent (Exime ${ }^{\circledR}$, Rocamed) that allows urine to bypass around the catheter. This device could provide minor discomfort, allowing complete urinary continence and spontaneous urination, while safely left in place for the necessary time (up to one month).

Rezūm treatment is not suitable for all patients. In particular, it has been cautiously contraindicated in patients with a urinary implant or penile prosthesis. The presence of recurrent or active urinary tract infection and/or urinary retention have been considered relative contraindications to treatment with Rezūm. Similarly, it is relatively not indicated in patients with previous prostate surgery, radiation treatment or focal therapy. Marion's disease or bladder neck stenosis is also a contraindication to this type of treatment. This study is not devoid of limitations. Although prospective in nature, it is a longitudinal cohort study with no control arm [25]. At present, there is a lack of data on long-term follow-up and the need for repeat or alternate procedures is unknown. However, even if more powered studies are awaited to adequately evaluate the retreatment rates, the impact on the quality of life and the pharmacoeconomics, Rezūm showed good outcomes on early follow-up. Apart from being randomized, future studies also need to be independent of funding or support from the industry or commercial company to avoid any potential bias in their conduct.

Author contributions GS: manuscript writing, project development, data collection. LC: manuscript editing, project development, data collection. GF: project development, data collection. DM: project development, data collection. GF: data collection. NC: data collection. FV: data collection. FV: data collection. SR: data collection. SC: data collection. BKS: manuscript editing. LV: data collection. AM: data analysis. MC: project development, supervisor.

\section{Declarations}

Conflicts of interest The authors declare that they have no conflict of interest.

Research involving human participants All procedures performed in this study involving human participants were in accordance with the ethical standards of the institutional and national research Committee and with the 1964 Helsinki declaration and its later amendments or comparable ethical standards.

Informed consent Informed consent was obtained from all individual participants included in the study.

\section{References}

1. Issa MM, Fenter TC, Black L et al (2006) An assessment of the diagnosed prevalence of diseases in men 50 years of age or older. Am J Manag Care 12:S83-89

2. Campbell FM, Walsh PC (2002) Urology, 8th edn. Saunders, pp 1297-1336

3. Lee SWH, Chan EMC, Lai YK (2017) The global burden of lower urinary tract symptoms suggestive of benign prostatic hyperplasia: A systematic review and meta-analysis. Sci Rep 7:7984. https:// doi.org/10.1038/s41598-017-06628-8

4. Fitzpatrick JM (2006) The natural history of benign prostatic hyperplasia. BJU Int 97(Suppl 2):3-6. https://doi.org/10.1111/j. 1464-410X.2006.06097.x (discussion 21-22)

5. Cindolo L, Pirozzi L, Fanizza C et al (2015) Drug adherence and clinical outcomes for patients under pharmacological therapy for lower urinary tract symptoms related to benign prostatic hyperplasia: population-based cohort study. Eur Urol 68:418-425. https:// doi.org/10.1016/j.eururo.2014.11.006

6. Cantrill CH, Zorn KC, Elterman DS, Gonzalez RR (2019) The Rezūm system-a minimally invasive water vapor thermal therapy for obstructive benign prostatic hyperplasia. Can J Urol 26:9787-9793

7. McVary KT, Rogers T, Roehrborn CG (2019) Rezūm water vapor thermal therapy for lower urinary tract symptoms associated with benign prostatic hyperplasia: 4-year results from randomized controlled Study. Urology 126:171-179. https://doi.org/10.1016/j. urology.2018.12.041

8. Suzuki T, Otsuka A, Ozono S (2016) Combination of intravesical prostatic protrusion and resistive index is useful to predict bladder outlet obstruction in patients with lower urinary tract symptoms suggestive of benign prostatic hyperplasia. Int J Urol 23:929-933. https://doi.org/10.1111/iju.13188

9. Green Z, Westwood J, Somani BK (2019) What's new in rezum: a transurethral water vapour therapy for BPH. Curr Urol Rep 20:39. https://doi.org/10.1007/s11934-019-0903-7

10. Zlotta AR, Giannakopoulos X, Maehlum O et al (2003) Longterm evaluation of transurethral needle ablation of the prostate (TUNA) for treatment of symptomatic benign prostatic hyperplasia: clinical outcome up to five years from three centers. Eur Urol 44:89-93. https://doi.org/10.1016/s0302-2838(03)00218-5

11. Mattiasson A, Wagrell L, Schelin S et al (2007) Five-year followup of feedback microwave thermotherapy versus TURP for clinical BPH: a prospective randomized multicenter study. Urology 69:91-96. https://doi.org/10.1016/j.urology.2006.08.1115 (discussion 96-97)

12. Mynderse LA, Roehrborn CG, Partin AW et al (2011) Results of a 5-year multicenter trial of a new generation cooled high energy transurethral microwave thermal therapy catheter for benign prostatic hyperplasia. J Urol 185:1804-1810. https://doi.org/10.1016/j. juro.2010.12.054

13. Roehrborn CG, Barkin J, Gange SN et al (2017) Five year results of the prospective randomized controlled prostatic urethral L.I.F.T. study. Can J Urol 24:8802-8813

14. Misrai V, Rijo E, Zorn KC et al (2019) Waterjet ablation therapy for treating benign prostatic obstruction in patients with small- to medium-size glands: 12-month results of the first French aquablation clinical registry. Eur Urol 76:667-675. https://doi.org/10. 1016/j.eururo.2019.06.024

15. Dixon CM, Rijo Cedano E, Mynderse LA, Larson TR (2015) Transurethral convective water vapor as a treatment for lower urinary tract symptomatology due to benign prostatic hyperplasia using the Rezūm( $\left({ }^{\circledR}\right)$ system: evaluation of acute ablative capabilities in the human prostate. Res Rep Urol 7:13-18. https://doi.org/ 10.2147/RRU.S74040 
16. Mynderse LA, Hanson D, Robb RA et al (2015) Rezūm system water vapor treatment for lower urinary tract symptoms/benign prostatic hyperplasia: validation of convective thermal energy transfer and characterization with magnetic resonance imaging and 3-dimensional renderings. Urology 86:122-127. https://doi. org/10.1016/j.urology.2015.03.021

17. McVary KT, Gange SN, Gittelman MC et al (2016) Minimally invasive prostate convective water vapor energy ablation: a multicenter, randomized, controlled study for the treatment of lower urinary tract symptoms secondary to benign prostatic hyperplasia. J Urol 195:1529-1538. https://doi.org/10.1016/j.juro.2015.10.181

18. Jones P, Rajkumar GN, Rai BP et al (2016) Medium-term outcomes of urolift (Minimum 12 Months Follow-up): evidence from a systematic review. Urology 97:20-24. https://doi.org/10.1016/j. urology.2016.05.003

19. Jones P, Rai BP, Nair R, Somani BK (2015) Current status of prostate artery embolization for lower urinary tract symptoms: review of world literature. Urology 86:676-681. https://doi.org/ 10.1016/j.urology.2015.05.011

20. Taktak S, Jones P, Haq A et al (2018) Aquablation: a novel and minimally invasive surgery for benign prostate enlargement. Ther Adv Urol 10:183-188. https://doi.org/10.1177/1756287218 760518

21. Roehrborn CG, Teplitsky S, Das AK (2019) Aquablation of the prostate: a review and update. Can J Urol 26:20-24
22. Johnston MJ, Noureldin M, Abdelmotagly Y et al (2020) Rezum water vapour therapy: promising early outcomes from the first UK series. BJU Int. https://doi.org/10.1111/bju.15203

23. Rocco B, Sighinolfi MC, Sandri M et al (2020) The dramatic COVID 19 outbreak in Italy is responsible of a huge drop of urological surgical activity: a multicenter observational study. BJU Int. https://doi.org/10.1111/bju.15149

24. Gratzke C, Barber N, Speakman MJ et al (2017) Prostatic urethral lift vs transurethral resection of the prostate: 2-year results of the BPH6 prospective, multicentre, randomized study. BJU Int 119:767-775. https://doi.org/10.1111/bju.13714

25. Reale G, Marchioni M, Altieri V et al (2020) Operative profile, safety and functional outcomes after GreenLight laser prostate surgery: results from a 12 months follow-up multicenter Italian cohort analyses. Minerva Urol Nefrol 72:622-628. https://doi.org/ 10.23736/S0393-2249.20.03597-3

Publisher's Note Springer Nature remains neutral with regard to jurisdictional claims in published maps and institutional affiliations. 